\title{
Randomised double blind placebo controlled trial of the effect of botulinum toxin on walking in cerebral palsy
}

\author{
T Ubhi, B B Bhakta, H L Ives, V Allgar, S H Roussounis
}

Academic Department of Paediatrics and Child Health, University of Leeds, UK

T Ubhi

Rheumatology and Rehabilitation Research Unit, University of Leeds B B Bhakta

Research \& Development Unit, St James's University Hospital, Leeds, UK V Allgar

Regional Child Development Centre, St James's University Hospital

H Ives

S Roussounis

Correspondence to: Dr T Ubhi, Molecular Medicine Unit, Clinical Sciences Building, St James's University Hospital, Beckett St, Leeds LS9 7TF, UK Medbsu@Leeds.ac.uk

Accepted 4 July 2000

\begin{abstract}
Background-Cerebral palsy is the commonest cause of severe physical disability in childhood. For many years treatment has centred on the use of physiotherapy and orthotics to overcome the problems of leg spasticity, which interferes with walking and can lead to limb deformity. Intramuscular botulinum toxin (BT-A) offers a targeted form of therapy to reduce spasticity in specific muscle groups.

Aims-To determine whether intramuscular BT-A can improve walking in children with cerebral palsy.

Design-Randomised, double blind, placebo controlled trial.

Methods-Forty patients with spastic diplegia or hemiplegia were enrolled. Twenty two received botulinum toxin and 18 received placebo. The primary outcome measure was video gait analysis and secondary outcome measures were gross motor function measure (GMFM), physiological cost index (PCI), and passive ankle dorsiflexion.
\end{abstract}

Results-Video gait analysis showed clinically and statistically significant improvement in initial foot contact following BT-A at six weeks and 12 weeks compared to placebo. Forty eight per cent of BT-A treated children showed clinical improvement in VGA compared to $17 \%$ of placebo treated children. The GMFM (walking dimension) showed a statistically significant improvement in favour of the botulinum toxin treated group. Changes in PCI and passive ankle dorsiflexion were not statistically significant.

Conclusion-The study gives further support to the use of intramuscular botulinum toxin type $A$ as an adjunct to conventional physiotherapy and orthoses to reduce spasticity and improve functional mobility in children with spastic diplegic or hemiplegic cerebral palsy. (Arch Dis Child 2000;83:481-487)

Keywords: cerebral palsy; botulinum toxin; gait analysis

Cerebral palsy (CP) is the commonest cause of severe physical disability in childhood, affecting 1 in 400 children. ${ }^{1}$ It consists of a heterogeneous group of motor disorders including spasticity, paresis, incoordination, and dystonia. Eighty per cent of these children have problems with walking as a result of lower limb spasticity, ${ }^{2}$ which can lead to severe contractures and limb deformity. Calf muscle spasticity is one major factor that can interfere with normal walking by preventing heel strike. $^{2}$ Conventional treatment is based around physiotherapy, ${ }^{3}{ }^{4}$ orthoses, and walking aids. ${ }^{4}$ However despite these therapies, many children require corrective surgery for deformity. The use of systemic antispasticity treatments such as baclofen, although effective, is limited by side effects such as drowsiness and generalised muscle weakness. ${ }^{5}$ Antispasticity treatments targeted at specific muscle groups can reduce the risk of generalised weakness. Destructive treatments using phenol nerve blocks, which can cause sensory loss, ${ }^{6}$ are inappropriate in the management of children who have a maturing nervous system. Botulinum toxin offers a targeted antispasticity treatment in children with cerebral palsy without causing loss of sensation. $^{7}$

Botulinum toxin is a potent neurotoxin produced by the anaerobic bacterium Clostridium botulinum. Seven serotypes (A-G) of botulinum toxin are known, with type A being available for clinical use. ${ }^{8}$ Botulinum toxin type A (BT-A) causes chemodenervation and muscle relaxation by disrupting acetylcholine release into the presynaptic cleft of the neuromuscular junction. ${ }^{9}$ BT-A has been used successfully in many diverse conditions where the clinical features arise from inappropriate muscle activity. ${ }^{10}{ }^{11}$ Side effects have been reported but are rare. ${ }^{12} 13$

Studies have shown that BT-A injection into the calf muscles of hereditary spastic mice allows normal longitudinal growth compared with untreated mice. ${ }^{14}$ This suggested that in children with CP, deformity might be reduced and mobility improved with BT-A treatment.

A number of open studies have reported improvement in walking following BT-A therapy. ${ }^{15-17}$ Cosgrove et al studied 26 children (some non-ambulant) with leg spasticity caused by cerebral palsy; 14 showed notable functional improvement following BT-A treatment. ${ }^{17}$ However, there have been few randomised placebo controlled studies. Koman et al originally reported improvement in the Physician's Rating Scale in six children randomised to receive BT-A compared with six children who received placebo. ${ }^{18}$ In their most recently published study, Koman et al showed improvement in gait function and partial denervation of the injected muscle as part of a larger multicentre trial. ${ }^{19}$ Sutherland et al reported significant improvement in ankle dorsiflexion while walking following BT-A treatment in a 


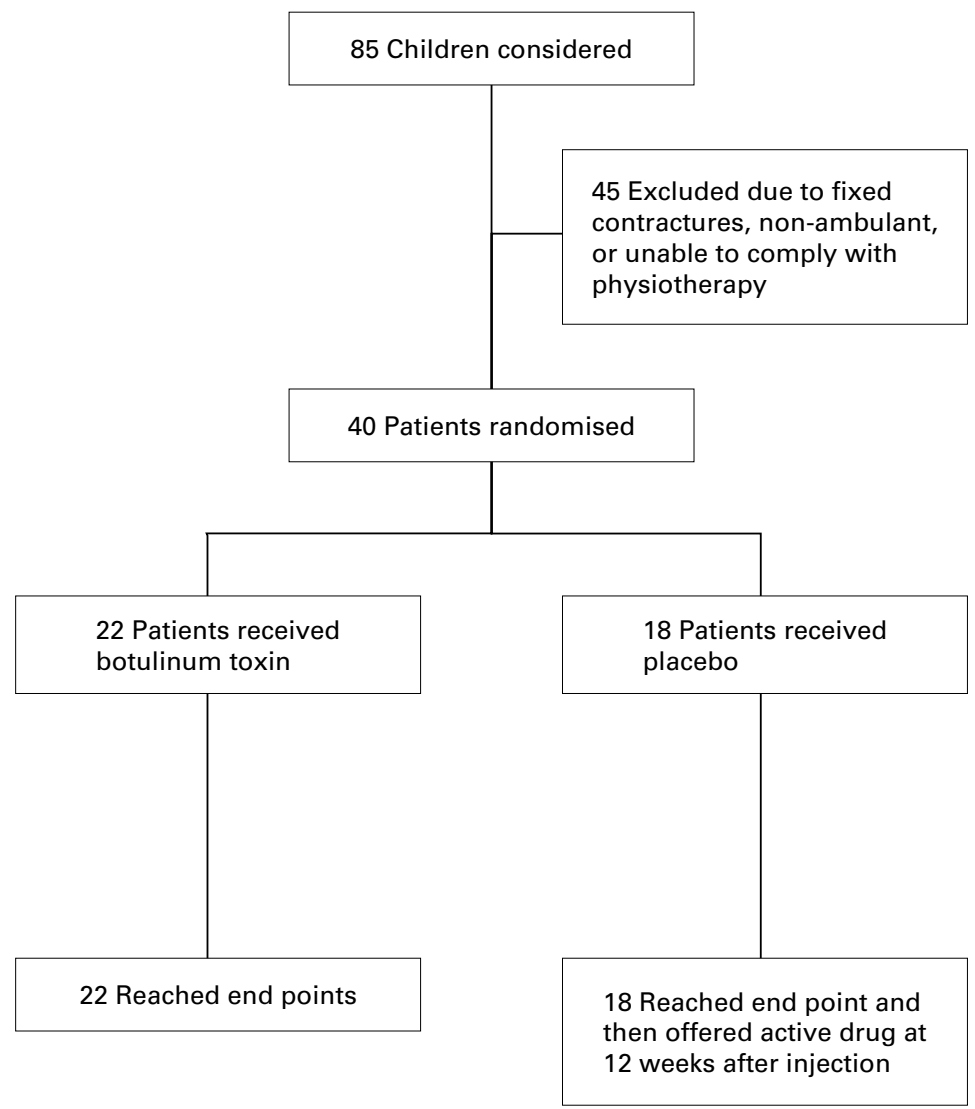

Figure 1 Trial profile.

controlled trial of 20 patients. ${ }^{20}$ Forssberg and Tedroff $^{21}$ highlighted the lack of controlled studies with appropriate functional outcome measures $^{22}$ and adequate power supporting the use of BT-A in children with CP. The UK Botulinum Toxin and Cerebral Palsy Working Party has also recently called for definitive

Table 1 Baseline demographic and clinical data

\begin{tabular}{|c|c|c|c|}
\hline & & Botulinum toxin $A$ & Placebo \\
\hline Patients & (n) & 22 & 18 \\
\hline Age at recruitment & Median (range) & $5.5(2.8-13.9)$ & $6.2(3.4-16.4)$ \\
\hline Gender ratio & $(\mathrm{F}: \mathrm{M})$ & $12: 10$ & $5: 13$ \\
\hline \multicolumn{4}{|l|}{ Type of cerebral palsy (n) } \\
\hline Hemiplegic & & 9 & 3 \\
\hline Diplegic & & 13 & 15 \\
\hline Initial foot contact & $(\mathrm{n}=$ legs $)$ & 34 & 33 \\
\hline Heel strike & (n) & 0 & 0 \\
\hline Flat foot strike & (n) & 1 & 1 \\
\hline Toe-heel strike & (n) & 3 & 2 \\
\hline Mild toe strike & (n) & 18 & 15 \\
\hline Marked toe strike & (n) & 12 & 15 \\
\hline GMFM (\%) & (n = patients $)$ & 21 & 15 \\
\hline Lying and rolling & Median (IQR) & $100(96.1-100)$ & $98.0(96.1-100)$ \\
\hline Sitting & Median (IQR) & $100(98.3-100)$ & $98.3(96.7-98.3)$ \\
\hline Crawling and kneeling & Median (IQR) & $97.6(90.5-100)$ & $92.9(78.6-97.6)$ \\
\hline Standing & Median (IQR) & $85.9(60.0-96.8)$ & $71.8(23.1-79.5)$ \\
\hline Walking and running & Median (IQR) & $69.4(26.4-86.5)$ & $54.2(18.1-79.2)$ \\
\hline Total & Median (IQR) & $89.0(74.5-96.3)$ & $84.0(62.0-90.0)$ \\
\hline Physiological cost index & Median (IQR) & $0.7(0.5,1.2)$ & $0.8(0.6,1.4)$ \\
\hline Passive ankle dorsiflexion & $\mathrm{n}$ & 20 & 16 \\
\hline Knee extended & Mean (SD) & $73.3(11.4)$ & $71.5(11.9)$ \\
\hline \multicolumn{4}{|l|}{ Dose of trial drug (U/kg) } \\
\hline Diplegic & mean (SD) & $24.8(3.2)$ & $24.2(5.4)$ \\
\hline Hemiplegic & mean (SD) & $16.4(4.0)$ & $14.4(1.6)$ \\
\hline \multicolumn{4}{|l|}{ Muscles injected } \\
\hline Gastrocnemius only & n (\%) & 0 & $5(15.1 \%)$ \\
\hline Gastrocnemius + soleus & $\mathrm{n}(\%)$ & $34(97.1 \%)$ & $26(78.8 \%)$ \\
\hline Gastrocnemius + hamstrings & $\mathrm{n}(\%)$ & $1(2.9 \%)$ & 0 \\
\hline $\begin{array}{l}\text { Gastrocnemius + soleus + } \\
\text { hamstrings }\end{array}$ & $\mathrm{n}(\%)$ & 0 & $2(6.1 \%)$ \\
\hline
\end{tabular}

studies to assess the impact of BT-A in childhood $\mathrm{CP}^{23}$

In this randomised, placebo controlled, double blind study we tested the hypothesis that reducing lower limb spasticity with BT-A would improve walking pattern.

\section{Materials and methods}

SUBJECTS

Forty children aged between 2 and 16 years with either spastic diplegic or hemiplegic CP were recruited from 85 consecutive referrals for BT-A therapy to the Yorkshire Regional Child Development Centre from September 1996 to March 1998 (fig 1). All could walk with or without walking aids. The children recruited had dynamic equinus with an inability to achieve heel strike because of lower limb spasticity predominantly affecting the calf muscles. None had received BT-A previously and all had received conventional treatment with physiotherapy and foot orthoses for a minimum of three months prior to treatment. This continued unchanged for the duration of the study. We excluded children who had fixed contractures or previous surgery to the lower limb.

The sample size and power was determined by changes in initial foot contact seen post botulinum toxin treatment in a previous pilot study. ${ }^{24}$ Fifty six children needed to be recruited into the study to give an $80 \%$ probability of detecting change at the $5 \%$ significance level. However, because of the stringency of the inclusion criteria, we recruited only 40 patients from 85 consecutive referrals. This gave a $70 \%$ probability of detecting change at a 5\% level.

The ethics committee approved this study. Parents received written and verbal information and gave written consent. All children were offered BT-A on an open basis if clinically indicated at the end of the study. Table 1 summarises the baseline demographic and clinical data.

\section{STUDY DESIGN}

We conducted this single centre study as a double blind, randomised, placebo controlled, parallel trial of botulinum toxin type A (Dysport) versus placebo. The medical statistics department independently produced an individual randomisation code ${ }^{25}$ for trial drug allocation with the hospital pharmacy undertaking masking, labelling, and dispensing. We conducted two pretreatment assessments two weeks apart to assess baseline variability of outcome measures. The trial drug was injected (by TU) after the second of these assessments. We made further assessments at two, six, and 12 weeks after injection.

All outcome measurements were performed by one experienced senior paediatric physiotherapist (HI) who was unaware of treatment allocation. Both parents and children were also masked to treatment allocation.

\section{INTERVENTION}

BT-A (Dysport) or placebo was made up to a standard concentration according to the manufacturer's instructions $(200 \mathrm{U} / \mathrm{ml}$ of $0.9 \%$ 
saline), resulting in a relatively small volume of injection and thus limiting pain from stretching the muscle capsule. The drug or placebo equivalent was then administered by intramuscular injection. The placebo contained all the excipients apart from toxin and was identical in appearance to the active drug. Trial drug at a dose equivalent to $25 \mathrm{U} / \mathrm{kg}$ body weight for diplegics (median age 5.9 years, range 3.416.4) and $15 \mathrm{U} / \mathrm{kg}$ for hemiplegics (median age 5.9 years, range 2.8-13.9) was administered using a 27 gauge needle into the gastrocnemius and soleus muscle groups. In addition to calf muscle injections, three patients received injections to the hamstrings because of excessive spasticity. Lateral and medial gastrocnemius muscles were injected with a mean dose of 99 U (BT-A) or $100 \mathrm{U}$ (placebo) each. Soleus was injected with a mean dose of $64 \mathrm{U}$ (BT-A) or 61 $\mathrm{U}$ (placebo). We identified the target muscles by clinical examination and used topical anaesthesia (EMLA cream) over the injection sites; oral midazolam at a dose of $0.5 \mathrm{mg} / \mathrm{kg}$ body weight was offered.

OUTCOME MEASURES

The primary outcome measure was video gait analysis (VGA). The secondary outcome measures were: gross motor function measure $(\mathrm{GMFM}),{ }^{26}$ passive ankle dorsiflexion, ${ }^{27}$ and physiological cost index (PCI). ${ }^{28}$

\section{Video gait analysis}

Observational gait analysis has been used in previous studies of botulinum toxin treatment in CP in the form of the Physician's Rating Scale (PRS). ${ }^{18}$ We used video gait analysis scoring (VGA), a form of observational gait analysis (table 2). This scale is similar to the foot contact subscale of the PRS although had been developed independently. The main difference between the VGA scale and the foot contact subscale of the PRS is that our scale had two grades of toe walking. As the scales were different, we analysed reproducibility of the VGA scoring used in this study. We performed standardised video gait recordings in both coronal and sagittal planes to determine initial foot contact during stance phase. Recordings were performed with the patient walking along a walkway without splints or footwear. All video recordings were independently compiled in a random order (with respect to patients, treatment, and time points) on to 15 viewing tapes by the audiovisual department. A senior paediatric physiotherapist, independent of the whole study and with no knowledge of the sequence of video recordings or treatment allocation, scored the 195 images (data not recorded on five). Another four paediatric physiotherapists not involved in the randomised control trial viewed all 15 tapes twice to determine the intra- and interobserver reproducibility of this scale. Each leg was scored separately. A change in one grade on this scale was deemed clinically significant. We summarised the VGA score for each child as follows: the walking pattern of the child was considered to have clinically improved if there was at least one grade improvement in either
Table 2 Grading of initial foot contact using video gait analysis

\begin{tabular}{lc}
\hline Initial foot contact & Score \\
\hline Normal heel strike & 0 \\
Flat foot & 1 \\
Toe then heel & 2 \\
Mild toe walking & 3 \\
Marked toe walking & 4 \\
\hline
\end{tabular}

one or both treated legs. If there was worsening of either leg following treatment the child was categorised as having deteriorated.

\section{Gross motor function measure}

The GMFM is a widely used assessment that has shown good reliability and validity as a measure of motor performance in children with $\mathrm{CP}^{26}$ and has been used in clinical trials of spasticity treatment. ${ }^{29}$ It is an observational test that has to be administered by a trained and accredited therapist or doctor, is expressed as a percentage (where $100 \%$ indicates maximum motor performance), and comprises five subcategories (lying and rolling; sitting; crawling and kneeling; standing; walking, running, and jumping). A change of $6 \%$ in the total score or within a dimension of the GMFM is considered to be clinically significant in children with cerebral palsy. Russell identified a $6 \%$ level as being clinically significant by comparing total and dimensional changes in the GMFM score with parents' and physiotherapists' rating of change of gross motor function. Therefore, we defined a successful outcome following treatment in this study as a change of at least $6 \%$ in the walking dimension of the GMFM.

\section{Passive ankle dorsiflexion}

Passive ankle dorsiflexion was measured using a protractor goniometer with the knee in maximum extension. The summary measurement used for children with diplegia was the mean of dorsiflexion measured in each leg and for children with hemiplegia, dorsiflexion was measured in the treated leg only.

\section{Physiological cost index}

PCI is an index of energy expenditure and may be used as a proxy for walking efficiency. ${ }^{28} \mathrm{We}$ asked children to perform four walks along a 6 metre walkway and recorded heart rate and time taken to complete the walks using a heart rate meter and chronograph. We calculated PCI using the formula: PCI $=\left(\mathrm{H}_{\mathrm{w}}-\mathrm{H}_{\mathrm{R}}\right) / \mathrm{S}$, where $\mathrm{H}_{\mathrm{W}}$ was the average heart rate throughout the walk in beats $/ \mathrm{min}, \mathrm{H}_{\mathrm{R}}$ was the heart rate at rest (prior to walking) in beats $/ \mathrm{min}$, and $\mathrm{S}$ was the speed of walking in metres/min. The mean PCI over the four walks was used for the analysis. A decrease in PCI value suggests increased walking efficiency.

\section{STATISTICAL ANALYSIS}

We judged efficacy of BT-A therapy on the change of scores between baseline and six weeks post injection, as the functional effects of BT-A were likely to be maximal at this stage. Further analysis was performed at two weeks and 12 weeks to judge speed of onset of effect and duration of effect. Summary data obtained 
Table 3 Change in summary VGA score

\begin{tabular}{|c|c|c|c|c|c|}
\hline & Worse & Same & Better & Total & Missing \\
\hline \multicolumn{6}{|c|}{ Baseline to week $2\left(\chi^{2}=3.06, p=0.08\right)$} \\
\hline Placebo & $4(24 \%)$ & $10(59 \%)$ & $3(18 \%)$ & 17 & 1 \\
\hline Botulinum toxin & $1(5 \%)$ & $12(71 \%)$ & $7(35 \%)$ & 20 & 2 \\
\hline \multicolumn{6}{|c|}{ Baseline to week $6\left(\gamma^{2}=5.42, p=0.02\right)$} \\
\hline Placebo & $4(22 \%)$ & $11(61 \%)$ & $3(17 \%)$ & 18 & 0 \\
\hline Botulinum toxin & $1(5 \%)$ & $10(48 \%)$ & $10(48 \%)$ & 21 & 1 \\
\hline \multicolumn{6}{|c|}{ Baseline to week $12\left(\chi^{2}=8.63, p=0.003\right)$} \\
\hline Placebo & $3(17 \%)$ & $13(72 \%)$ & $2(11 \%)$ & 18 & 0 \\
\hline Botulinum toxin & 0 & $10(50 \%)$ & $10(50 \%)$ & 20 & 2 \\
\hline
\end{tabular}

from the two pretreatment assessments are used to describe baseline variability of the outcome measures. $\chi^{2}$ for trend and the MannWhitney test were used to analyse changes in GMFM and VGA. Analysis of covariance was used to analyse changes in goniometry and PCI. The 5\% significance level was used throughout the analysis. The $\kappa$ statistic and the intraclass correlation coefficient was used to describe inter- and intraobserver reproducibility of the VGA scoring system respectively.

\section{Results}

Twenty two children received BT-A (median age 5.5 years, range $2.8-13.9$ ) and 18 received placebo (median age 6.2 years, range 3.4 16.4). One child in the BT-A group was taking regular oral baclofen. All children received topical anaesthesia and nine received oral sedation (seven in the BT-A group and two in the placebo group).

VIDEO GAIT ANALYSIS

We observed significant improvement in initial foot contact at six weeks $(p=0.02)$ and 12 weeks $(p=0.003)$ following botulinum toxin compared to placebo (table 3 ). We found no significant difference between placebo and botulinum toxin treatment at two weeks. Video gait analysis showed moderate to good interobserver reliability with an overall $\kappa$ score for the left leg of 0.65 and right leg 0.61 . Intraobserver reproducibility was also high (0.86 left, 0.66 right).

GROSS MOTOR FUNCTION MEASURE

The GMFM in the walking dimension at week 12 showed a statistically significant difference in the proportions of those who showed clinically significant improvement (greater than $6 \%$ change in the GMFM score). Thirty seven per cent (7/19) of patients (mean improvement $9.7 \%$ ) treated with BT-A showed clinically significant improvement compared with $7 \%$ $(1 / 15)$ in the placebo group $\left(\chi^{2}=4.24\right.$, $\mathrm{p}=0.04$,$) . Three of seven patients who had$ improved with botulinum toxin had hemiplegia. Six patients (mean age 5 years, range 3.69.4) failed to complete the GMFM because of a lack of cooperation. Significant differences were not seen in the other dimensions or in the total GMFM score. There were no significant differences between treatment groups at weeks 2 and 6.

PASSIVE ANKLE DORSIFLEXION

There were no significant differences in changes of passive ankle dorsiflexion between botulinum toxin and placebo at week 2 (BT-A mean change $2.3,95 \%$ confidence interval (CI) $0.6,4.1$; placebo mean change $2.8,95 \%$ CI $-0.4,6.0$ ), week 6 (BT-A mean change 3.8, $95 \%$ CI 1.4, 6.0; placebo mean change 1.7, $95 \% \mathrm{CI}-2.9,6.2$ ), and week 12 (BT-A mean change $2.2,95 \%$ CI $-1.4,5.9$; placebo mean change $-0.3,95 \% \mathrm{CI}-3.3,3.8)$.

\section{PHYSIOLOGICAL COST INDEX}

At baseline the median PCI in the BT-A group was $0.7(0.5,1.2)$ beats per metre and $0.8(0.6$, 1.4) beats per metre in the placebo group. At six weeks the PCI was $0.8(0.6,1.1)$ beats per metre in the BT-A group and $0.9(0.6,1.6)$ beats per metre in the placebo group. These differences were not statistically significant. No significant differences were seen at weeks 2 and 12.

\section{ADVERSE EVENTS}

Six children treated with BT-A reported adverse events: significant post injection calf pain requiring simple analgesia in two, increased frequency of falls within the first two weeks after injection in two, wheeziness in one, and seizures in one (who was, however, known to be liable to seizures). One child treated with placebo vomited after injection. We observed no evidence of excessive muscle weakness (for example, crouch gait) following trial drug administration. All adverse events were self limiting.

\section{Discussion}

This is the first large, well controlled, single centre, randomised study that has shown an improvement in walking pattern in children with cerebral palsy using BT-A targeted at the calf muscles. Improvement was above that found with physiotherapy and splintage alone and was maintained to 12 weeks following injection. Adverse events relating to botulinum toxin were mild and transient, in keeping with previous published reports. ${ }^{16} 17$ Several outcome measures were used to assess impact of BT-A in these children. Of these, video gait analysis was quick and easy to perform and proved to be a robust objective measure of foot placement during walking. It also showed good intra- and interobserver reliability and has good face validity. Therefore, it could easily be used in the outpatient setting where current assessment tools are limited in their applicability. The Physician's Rating Scale and gross motor function measure are both time consuming measures and the latter must also be done by a trained therapist or doctor and typically takes an hour to complete. Therefore, although it is useful as a research tool, it would be difficult to use in a paediatric clinic. In this context the VGA offers a simple and quick method of assessing and monitoring children following BT-A injections. It is, however, unable to discriminate the quality of movement in the same way as more advanced techniques such as $3 \mathrm{D}$ gait analysis. It should be noted that only $50 \%$ of those treated with BT-A showed significant clinical improvement. Secondary analysis of these cases did not reveal the cause 
of non-response in terms of age at treatment or type of cerebral palsy.

This study highlights the difficulties in obtaining a homogeneous group of children with spastic cerebral palsy from a single centre within a study period of 18 months. Exclusions related to non-ambulant status, fixed equinus deformity, and predominantly hip adductor and hamstring spasticity. Only three suitable children declined to participate.

The only other published randomised controlled trials to date are by Koman and colleagues $^{1819}$ and Sutherland et al. ${ }^{20}$ Koman and colleagues ${ }^{19}$ reported an improvement in walking in children with cerebral palsy as part of a multicentre international study comparing botulinum toxin against placebo. They used observational gait analysis, ankle dorsiflexion, and nerve conduction studies as their outcome measures. In that study, gait pattern and "ankle position components" showed the most significant changes after botulinum toxin injections. The response rate for improvement of equinus foot position in $50-60 \%$ of patients without significant morbidity is similar to our own findings. The report published by Sutherland and colleagues analysed the outcome of 20 children who were randomised to receive placebo or BT-A. ${ }^{20}$ Patients had been given two treatment courses four weeks apart (placebo or BT-A). It was unclear whether this treatment had been given in addition to physiotherapy or splintage. Analysis of their results was confined to the right leg only in patients who had both legs treated $(n=8)$. In two children with diplegia, only the left leg alone was injected and in those cases (as with hemiplegics) the treated leg was used for analysis. Significant improvement in ankle dorsiflexion was identified during walking (as measured by three dimension 5 camera Vicon motion measurement system) in children who had received BT-A. No changes in passive ankle dorsiflexion were found. These workers also highlighted the lack of relevant objective outcome measures.

Spasticity impairs gait pattern and considerably increases energy expenditure. ${ }^{30}$ This is confirmed by the baseline PCI values in our study (table 1). The normal PCI in children is $0.4-0.6$ beats/metre ${ }^{28}$ whereas baseline values were much greater in both the placebo and BT-A treated groups. Massin and Allington ${ }^{31}$ used exercise testing (which estimates energy cost) to measure the functional impact of BT-A in children with cerebral palsy. BT-A was found to reduce the energy cost of movement and improved the endurance of spastic muscles. This contrasts with our finding of no significant difference in the PCI. Unfortunately, the PCI proved technically difficult to do in the younger children and there was also large variability in the recorded heart rate during the walks. Boyd and $\mathrm{Graham}^{32}$ have recently questioned the validity of the PCI as an outcome measure, suggesting that the high degree of variability seen in PCI measurement invalidates it in children with cerebral palsy.

The dose of BT-A (Dysport) used was derived from our pilot study. ${ }^{24} \mathrm{~A}$ dose of 25 U/kg BT-A in children with spastic diplegia and
$15 \mathrm{U} / \mathrm{kg}$ in children with spastic hemiplegia proved effective and safe and was in keeping with the dose range of Dysport used by other workers in the field at the inception of our study. Recent studies ${ }^{33}$ have also used similar doses (20-25 U/kg). Wissel et al, ${ }^{34}$ in a smaller study, compared considerably higher equivalent doses of Botox with low dose in various spastic muscles in the leg. They found a dose dependent response in knee flexor muscle tone, walking speed, and stride length without increase in systemic effects. However, they found no difference in the child's or parents' subjective rating of improvement.

Oral midazolam was useful when injecting trial drug in those patients who were young. Apart from its sedative effect, its additional amnesic properties have been found to allow greater cooperation with repeat injections.

Six children reported side effects following botulinum toxin injection: all were short lived and none required medical intervention. Major adverse effects are uncommon although minor side effects such as flu like symptoms following injection are recognised. No cases of systemic botulism as a result of BT-A injections have been reported. ${ }^{23}$ Although antibodies to BT-A are produced in adults, the incidence and significance in the paediatric population has not been established. The presence of such antibodies may lead to drug resistance developing and would thus support avoiding short injection intervals. ${ }^{23}$

Clinically significant changes in overall mobility (as identified by VGA and GMFM) occurred at weeks 6 and 12 post BT-A. Eames and colleagues ${ }^{33}$ studied the change in gastrocnemius muscle length following BT-A injection in 39 children and correlated the pretreatment dynamic component with the magnitude and duration of the response. They found greatest change in muscle length two weeks following BT-A. Similarly Cosgrove and colleagues ${ }^{17}$ reported reduction in calf spasticity two weeks after BT-A injection. These findings contrast with our findings of functional improvement at weeks 6 and 12 rather than at two weeks post treatment. One explanation for this difference is that the child has to adapt to new mechanical forces in the legs following muscle relaxation with BT-A. Corry and colleagues ${ }^{35}$ who compared stretching casts with BT-A in 20 children with an equinus deformity did, however, find an improvement in initial foot contact at weeks 2 and 12 .

Although we anticipated that reduction in calf muscle spasticity would improve passive ankle dorsiflexion as shown in open studies, ${ }^{15-17}$ no evidence of this was found from our study, confirming earlier findings of Sutherland et al. ${ }^{20}$ This raises the question of the relation between measurement of passive movement and changes observed during walking. One possible explanation of the lack of effect of BT-A on passive movement, but its positive effect on foot placement is that BT-A effects the "dynamic" spastic component as opposed to the range of passive movement which encapsulates resistance produced by muscle or joint connective tissue. ${ }^{32}$ 
The GMFM is a valid measure of function in children with cerebral palsy and is also sensitive to change over time. ${ }^{22} 26$ There was a clinically significant improvement in seven patients following BT-A compared with a single placebo case within the walking dimension of the GMFM at week 12. This was despite the fact that the BT-A group had a higher baseline GMFM score compared with the placebo group. There was no significant change in the other four dimensions following treatment, thus explaining the lack of significant change in the total GMFM score. This did not surprise us as all the children enrolled were ambulant and were expected and indeed did score highly on all dimensions apart from walking at baseline. Our study did not show differences in improvement in GMFM between children with diplegia compared to those with hemiplegia following BT-A. Although the GMFM is a recommended measure of motor performance in children with cerebral palsy, there is a lack of its use as an outcome measure within controlled trials. Despite the fact that it has been used as an outcome measure in long term studies after surgical treatments, ${ }^{29}{ }^{36}$ there have been no previous controlled studies using GMFM to measure outcome following BT-A treatment.

Botulinum toxin is not intended as a replacement for physiotherapy or orthotics, instead it should be viewed as an adjunct to current therapeutic strategies. Indeed there is an argument for increased physical therapy and optimal splintage following BT-A to capitalise on the muscle relaxation effect, allowing muscle stretching exercises to be performed more easily. Intensive physiotherapy treatment blocks following BT-A injection, to maximise muscle lengthening and thus provide improved long term benefit, have now become our standard management.

Although our study has gone some way to address the criticisms raised by Forssberg and Tedroff, ${ }^{21}$ it is limited in terms of its power. This was because of the difficulty in recruiting suitable children who fulfilled the inclusion criteria, even over a long period of time.

A number of important questions still need to be answered. These include the optimal age to start treatment, time interval between repeated treatments, differences between diplegics and hemiplegics, effect on long term mobility, perioperative analgesic effect, cost effectiveness, ${ }^{37}$ and long term adverse effects of repeated treatment. Secondary analysis of our data did not show a difference in outcome between diplegics and hemiplegics. Neither did it show an age effect. However, our clinical experience (not supported by the current study) suggests that treatment at an early age before the development of contractures produces better results and may prevent deformity, thus giving long term benefit. This is supported by the findings of Wissel and colleagues $^{34}$ who identified a differential effect of BT-A on children above and below age 7 . Eames and colleagues ${ }^{33}$ have suggested that the age effect may be because of a change of the child's problems from a dynamic to a fixed defect over time as contractures develop.
In conclusion, we have shown that BT-A is safe and easy to administer, can be given as an outpatient procedure, and results in an improvement in walking. It offers an alternative to surgical intervention and our study supports its use in children with cerebral palsy.

The authors would like to thank the Northern \& Yorkshire Health Authority and the Special Trustees at St James's University Hospital for funding this project. We are grateful to Mrs Mary Harrison for independently analysing the video gait data, the Audiovisual Department at the University of Leeds for randomising the video data, and Dr Alastair Cozens for help with study design. Our thanks go to Mrs Iris Maher for typing the script and Mrs Kathryn Ackroyd for nursing assistance. The authors would also like to thank IPSEN Ltd for supplying botulinum toxin (Dysport) and placebo.

1 Hagberg B, Hagberg G. Origins of cerebral palsy. In: David J, ed. Recent advances in paediatrics, No. 11. London: Churchill Livingston, 1999:67-83.

2 Gage JR. Clinics in developmental medicine, No. 121. Gait analysis in cerebral palsy. Oxford: Mackeith Press, 1991.

3 Smith MF. Advances in the management of cerebral palsy. In: David TJ, ed. Recent advances in paediatrics, No. 17 London: Churchill Livingston, 1999:129-40.

4 Hainsworth F, Harrison MJ, Sheldon TA, Roussounis SH. A preliminary evaluation of ankle orthoses in the management of children with cerebral palsy. Dev Med Child Neurol 1997;39:243-7.

5 Diamond M. Rehabilitation strategies for the child with cerebral palsy. Pediatr Ann 1996;15:230-6.

6 Botte MJ, Abrams RA, Bodinefowler SC. Treatment of acquired muscle spasticity using phenol peripheral nerve blocks. Orthopedics 1995;18:151-9.

7 Neville B. Botulinum toxin in the cerebral palsies. BMF 1994;309:1526-7.

8 Hambleton P, Moore AP. Botulinum neurotoxins: origin, structure, molecular actions and antibodies. In: Moore AP, ed. Handbook of botulinum toxin treatment. Oxford: Blackwell Science Ltd, 1995:16-27.

9 Blasi J, Chapman ER, Link E, et al. Botulinum neurotoxin A selectively cleaves the synaptic protein SNAP-25. Nature 1993;365:160-3.

10 Jankovic J, Brin MF. Therapeutic uses of botulinum toxin. $N$ Engl f Med 1991;324:1186-94.

11 O'Brien CF. Overview of clinical trials and published reports of botulinum toxin for spasticity. Eur $\mathcal{f}$ Neurol 1997;4:S11-13.

12 Tsui JKC, Eisen A, Stoessl AJ, Calne S, Calne DB. Double blind placebo controlled trial of botulinum toxin in spasmodic torticollis. Lancet 1986;2:245-7.

13 Erbguth FJ. Systemic effects of local botulinum toxin injections unmasks subclinical Lambert-Eaton myasthenic syndrome. F Neurol Neurosurg Psychiatry 1993;56:1235-6.

14 Cosgrove AP, Graham HK. Botulinum toxin A prevents the development of contractures in the hereditary spastic mouse. Dev Med Child Neurol 1994;36:379-85.

15 Koman LA, Mooney JF III, Smith B, Goodman A, Mulvaney T. Management of spasticity in cerebral palsy with botulinum A toxin. Preliminary investigation. $\mathcal{f}$ Pediatr Orthop 1993;13:489-95.

16 Wong V. Use of botulinum toxin in 17 children with spastic cerebral palsy. Pediatr Neurol 1998;18:124-31.

17 Cosgrove AP, Corry I, Graham HK. Botulinum toxin in the management of the lower limb in cerebral palsy. Dev Med Child Neurol 1994;36:386-96.

18 Koman LA, Mooney JF III, Smith BP, Goodman A, Mulvaney T. Management of spasticity in cerebral palsy with botulinum A toxin; report of a preliminary, randomised, double-blind trial. F Pediatr Orthop 1994;14:299-303.

19 Koman LA, Mooney JF, Paterson Smith B, Walker F, Leon $\mathrm{JM}$, and the BOTOX study group. Botulinum toxin type A neuromuscular blockade in the treatment of lower extremity spasticity in cerebral palsy: A randomised double-blind, placebo-controlled trial. F Pediatr Orthop 2000;20:108-15.

20 Sutherland DH, Kaufman KR, Wyatt MP, Chambers HG, Mubarak SJ. Double-blind study of botulinum A toxin injections into the gastrocnemius muscle in patients with injections into the gastrocnemius muscle in

21 Forssberg H, Tedroff KB. Botulinum toxin treatment in cerebral palsy: intervention with poor evaluation? Dev Med Child Neurol 1997;39:635-40.

22 Campbell SK. Quantifying the effects of interventions for movement disorders resulting from cerebral palsy. $\mathcal{f}$ Child Neurol 1996;11:S61-70.

23 Carr LJ, Cosgrove AP, Gringras P, Neville BGR. Position paper on the use of botulinum toxin in cerebral palsy. Arch Dis Child 1998;79:271-3.

24 Roussounis SR, Bhakta B, Harrison MH, Ives H, Ubhi BS. Botulinum toxin in cerebral palsy; experiences from an open study. Dev Med Child Neurol 1998;40(suppl 79):30.

5 Pocock SJ. Clinical trials: a practical approach. New York: J Wiley \& Sons, 1983:74.

26 Russell DJ, Rosenbaum PL, Cadman DT, Gowland C, Hardy S, Jarvis S. The gross motor function measure: a Hardy S, Jarvis S. The gross motor function measure: a
means to evaluate the effects of physical therapy. Dev Med Child Neurol 1989;31:341-52. 
27 Stuberg WA, Fuchs RH, Miedaner JA. Reliability of goniometric measurements of children with cerebral palsy. $D e v$ Med Child Neurol 1988;30:657-66.

28 Butler P, Engelbrecht M, Major RE, Tait JH, Stallard J, Patrick JH. Physiological cost index of walking for norma children and its use as an indicator of physical handicap. Dev Med Child Neurol 1984;26:607-12.

29 Wright FV, Sheil EM, Drake JM, Wedge JH, Naumann S. Evaluation of selective dorsal rhizotomy for the reduction of spasticity in cerebral palsy: a randomised controlled trial. Dev Med Child Neurol 1998;40:239-47.

30 Rose J, Gamble JG, Lee J, et al. The energy expenditure index: a method to quantitate and compare walking energy expenditure for children and adolescents. 7 Pediatr Orthop 1991;11:571-8.

31 Massin M, Allington N. Role of exercise testing in the functional assessment of cerebral palsy children after botulinum toxin injection. F Pediatr Orthop 1999;19:362-5.

32 Boyd R, Graham HK. Botulinum toxin A in the management of children with cerebral palsy: indications and ment of children with cerebral palsy:
33 Eames NWA, Baker R, Hill N, Graham K, Taylor T, Cosgrove A. The effect of botulinum toxin A on gastrocneCosgrove A. The effect of botulinum toxin A on gastrocneChild Neurol 1999;41:226-32.

34 Wissel J, Heinen F, Schenkel A, Doll B, Ebersbach G, Muller $\mathrm{J}$, Poewe W. Botulinum toxin A in the management of spastic gait disorders in children and young adults with cerebral palsy: a randomised double blind study of high dose verses low dose treatment. Neuropediatrics 1999;30:120-4

35 Corry IS, Cosgrove AP, Duffy CM, McNeill S, Taylor TC, Graham HK. Botulinum toxin A compared with stretching casts in the treatment of spastic equinus: a randomised prospective trial. $\mathcal{F}$ Pediatr Orthop 1998;18:304-11.

36 Steinbok P, Reiner A, Kestle JR. Therapeutic electrical stimulation following selective posterior rhizotomy in children with spastic diplegic cerebral palsy: a randomised trial. Dev Med Child Neurol 1997;39:515-20.

37 Wallesch CW, Maes E, Lecomte P, Bartels C. Cost effectiveness of bW, Maes E, Lecomte P, Bartels C. Cost effectivespasticity following stroke: a German perspective. Eur $\mathcal{F}$ Neurol 1997:4:S53-7.

\section{The uncertainty principle}

For a randomised trial to be ethically valid there should be uncertainty about which of the treatments concerned is better. If there is complete uncertainty, there should be an equal chance of either outcome and half of all trials should favour the new treatment and half the control treatment. Deviation from this equipoise would suggest either bias or poor quality trials. (This argument is, of course, an absolutist argument in that it insists on complete uncertainty. You might, perhaps, expect some loading in favour of new treatments as such treatments are often developed because there are good theoretical reasons to think that they might be better than standard treatments.) A report from the USA (Benjamin Djulbegovic and colleagues. Lancet 2000;356:635-8) has shown a heavy loading in favour of new treatments in trials sponsored by commercial firms.

They analysed 136 published randomised trials of treatment for multiple myeloma. Ninety-five were funded by government or other non-profit organisations and 35 had commercial funding (six were of unknown funding). The overall trial quality was poor but commercially funded trials were slightly better. In general, equipoise was maintained, $56 \%$ of trials favouring the tested treatment and $44 \%$ the control treatment. However, there was a distinct difference between commercially and non-commercially funded trials, with $74 \%$ of the former and $53 \%$ of the latter favouring the tested treatment. Trials funded by non-profit organisations were much more likely to have active rather than placebo or untreated controls.

Trials sponsored by the pharmaceutical industry are usually well conducted, but may have inferior control groups and may seem biased in favour of the tested treatment. But the industry will no doubt argue that their products are designed so carefully that by the time they reach the stage of clinical trials they are likely to be effective. However, although on theoretical grounds a new drug may have advantages over previous drugs, pharmaceutical companies must arrange randomised trials to prove it. That does not seem to me to involve any compromise of ethical principles. Perhaps in unusual circumstances, when there are very good grounds for suspecting that a new treatment will be effective, there is no effective treatment already available, and the condition being treated is very serious, it might be ethical to perform a non-randomised trial with historical controls, but randomised trials will surely remain the general rule.

ARCHIVIST 